\title{
Fast Low Voltage Ferroelectric Liquid Crystal for Field Sequential Color Display
}

\author{
Qi Guo, Fan Fan, Anatoli Murauski, Lishuang Yao, Vladimir Chigrinov \\ Center for Display Research, Department of Electronic and Computer Engineering, Hong Kong University of \\ Science and Technology, Clear Water Bay, Kowloon, Hong Kong
}

Received February 13, 2011; accepted March 29, 2011; published March 31, 2011

\begin{abstract}
In this paper, a fast responded ferroelectric liquid crystal (FLC) driven with low voltage is achieved. The switching time and contrast ratio of two different FLC materials are investigated with the temperature range from room temperature to $80^{\circ} \mathrm{C}$. The switching time of $50 \mu \mathrm{s}$ and contrast of $300: 1$ are obtained with FLC595 with $5 \mathrm{~V}$ voltage at room temperature, and switching time decreases with higher temperature. Compared to FLC595, FD4004N has a larger temperature range and different optimal temperature of about $40^{\circ} \mathrm{C}$, at which the contrast is $360: 1$. Proposed ferroelectric liquid crystals show excellent properties which is highly promising for future applications.
\end{abstract}

Pico projectors with their large potential market draw most attention because they could be embedded into mobile-phone handsets and have an available market of 1 billion units annually. This market will require a pico projector of high performance with low cost and consuming very little power [1]. We believe the field sequential color (FSC) is the only feasible route to pico projectors that satisfies these requirements [2, 3]. Scanned laser projectors are appealing, but reducing their image speckle to acceptable levels may be more challenging than first thought [4]. Projection engines based on threepanel color-splitting architecture offer high optical efficiency, but their cost, size, and complexity weigh against their use in pico projectors [5] Light absorption by color filters reduces optical efficiency of color-filterarray (CFA) displays compared to FSC displays [6].

The target response time for FSC displays should be less than $1 \mathrm{~ms}(240 \mathrm{~Hz}$ frame frequency) to provide high resolution, low power consumption and extended color gamut [7]. A fast switching ferroelectric liquid crystal display (FLCD) is a good candidate for the new generation of FSC LCD [8, 9]. Fast FLC switching enables high-quality color-sequential images without color-filters, at the same time realizes low-power consumption. In this paper, two suitable FLC materials are investigated, and their electro-optical responses are provided

The electro-optical measurements were performed in an automatic regime. For this aim a measurement device was built, whose principal scheme is shown in Fig. 1. The basic element of this experimental set-up is a computer data acquisition (DAQ) board NIPCI 6251 from National Instruments. This board has two analog outputs and 16 analog inputs, the minimal registration time is $1 \mu \mathrm{s}$. A Wideband Power Amplifier KH model 7600 from KrohnHite Corporation with amplification coefficients $* 5$ and *25 times was used. It enables output continuously variable voltage up to $\pm 250 \mathrm{~V}[10]$. A photo-detector was connected to the input board plate for optical measurements.

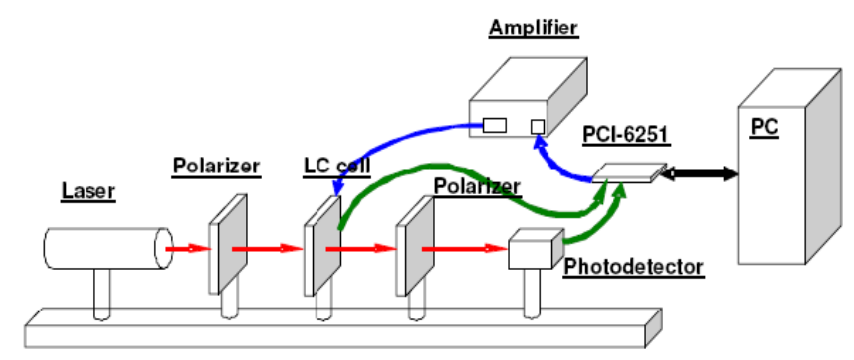

Fig. 1. Experimental set-up for electro-optical measurements.

The software for the experimental set-up has built-in functions for analog output-input. The program has three functional blocks which make the operation with the setup very effective [11]. The first block is a programmable generator that realizes any form of signal with a duration of 2000 points. The duration of one point can be set from $1 \mu$ s to $1 \mathrm{~s}$. The second block is a measuring block, which saves 4000 values of the input voltage with a step from $1 \mu \mathrm{s}$ to $1 \mathrm{~s}$. The operation of the first and the second blocks is synchronized inside the DAQ board and cannot be disturbed by computer interruptions. The third block is used to accumulate the experimental data.

*E-mail: qguo@ust.hk 


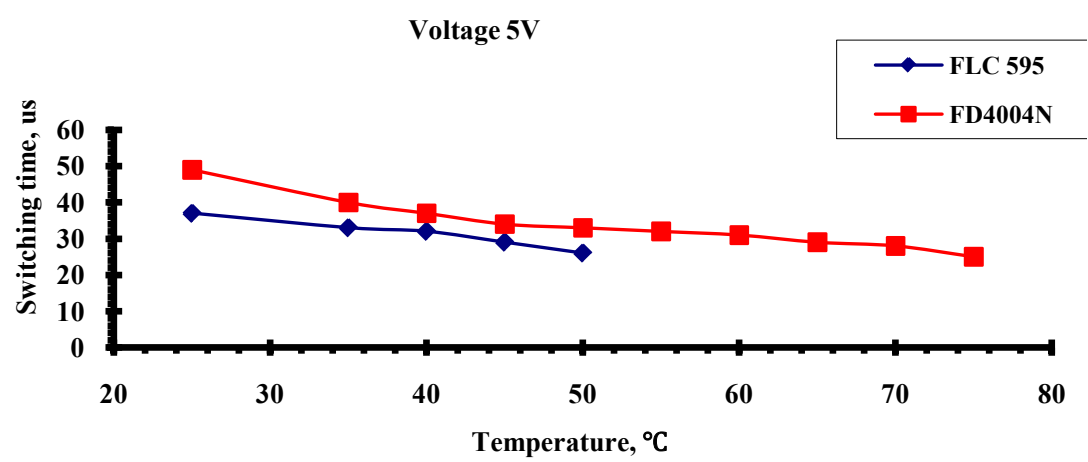

Fig.2. Dependence of switching time on different temperature.
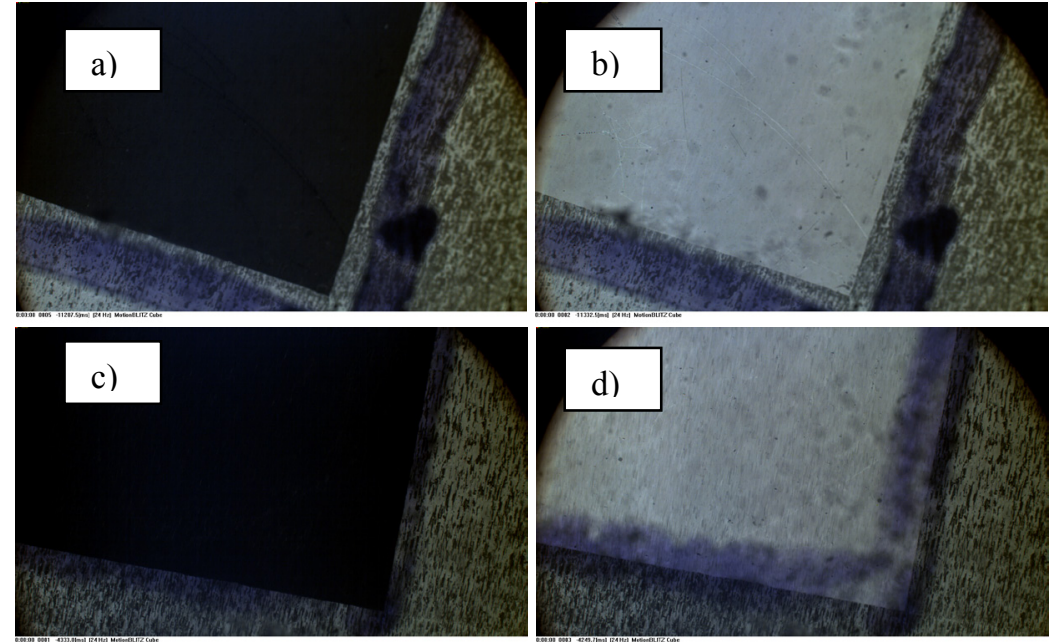

Fig. 3. Bright state and dark state of FD4004N and FLC595 (a: dark state of FD4004N; b: bright state of FD4004N; c: dark state of FLC595; d: bright state of FLC595).

Two FLC mixtures were used for experiments, in which light scattering in the visible spectral range is excluded completely. One of the FLC mixtures is FLC-595 from the P.N. Lebedev (Physical Institute in Moscow), the other one is FD4004N from DIC Ltd. Polyimide PI3744 layer rubbed with a piece of lawn was used for aligning FLC molecules. We used the cells with the size of $13 \times 17 \mathrm{~mm}^{2}$, electrodes area $5 \times 5 \mathrm{~mm}^{2}$, and the cell gap $0.8 \mu \mathrm{m}$.

The results of the switching time of FD4004N and FLC595 dependence on temperature are shown in Fig. 2.

The switching time is defined as $\tau_{0.1-0.9}$ with a driving signal of plus minus voltage with $500 \mathrm{~Hz}$ applied. The electro-optical response is not sensitive to the polarity of driving voltage, which is similar to liquid crystalline cells based on nematic liquid crystals but with much less response time. The switching time of less than $40 \mu \mathrm{s}$ is obtained with FLC595 on 5V voltage at room temperature, and the switching time even decreases with a higher temperature. Compared to FLC595, FD4004N has a larger temperature range but slightly longer switching time, which is also sufficient for field-sequential-color display.

Many methods are used to obtain a defect-free alignment of surface stabilized FLC [12, 13]. A zigzag defect-free SSFLC reported by Furue et al. and fabricated using a developed polymide RN 1199 (Nissan Chemical) with a small pretilt angle, which is used to smooth the alignment surface [12]. Kurihara et al. implemented an estimated critical value of the azimuthal anchoring energy with which a transition from the $\mathrm{C} 1$ to $\mathrm{C} 2$ uniform state occurs [13]. However, the defects in FD4004N cells exit just after cooling down. The FLC layer undergoes a transition from the $\mathrm{SmA}$ phase to $\mathrm{SmC}^{*}$, which involves zigzag defects [7]. After the electrical treatment with DC voltage on very low frequency of the cell, the FLC layer will extend to a uniform "bookshelf" structure without any defects. Figure 3 shows the bright state and dark state of cells with FD4004N and FLC595 after electrical treatment. 


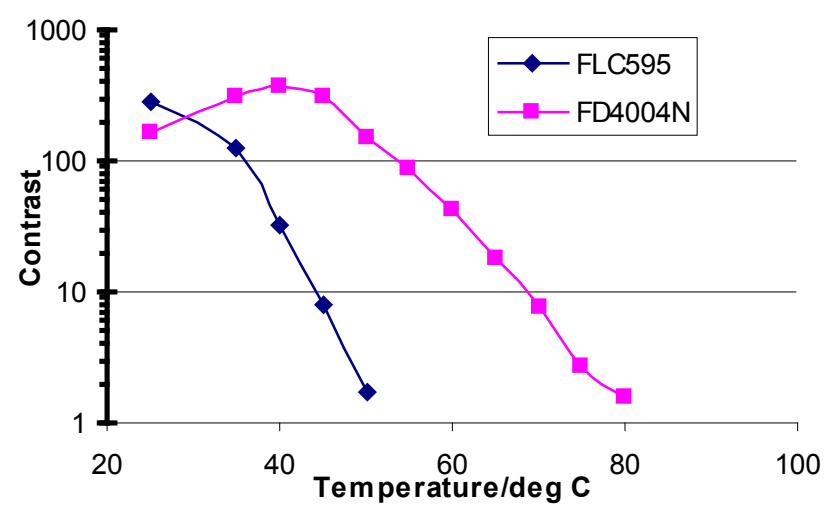

Fig. 4. Dependence of contrast on different temperature.

The contrast ratio of FD4004N and FLC595 are investigated with the temperature range from room temperature to $80^{\circ} \mathrm{C}$, shown in Fig. 4. Contrast of $300: 1$ are obtained with FLC595 on 5V voltage at room temperature. FD4004N has a larger temperature range and a different optimal temperature of about $40^{\circ} \mathrm{C}$, at which the contrast is $360: 1$.

In summary, we have demonstrated a method to achieve fast response with a low voltage with a ferroelectric liquid crystal. The electro-optical response of two materials FD4004N and FLC595 is investigated. More than 300 contrast ratios can be obtained. The switching time of less than $50 \mu \mathrm{s}$ can be obtained with these two materials, and the switching times are relatively stable within temperature ranges. This method is very attractive for the production of a field-sequential-color pico projector.

This work is supported by HKUST grants CERG 612310 and CERG 612208

\section{References}

[1] M.A. Handschy, B.F. Spenner, Inf. display 2412 (2008).

[2] M.J. O’Callaghan, R. Ferguson, R. Vohra, W. Thurmes, A.W. Harant, C.S. Pecinovsky, Y.Q. Zhang, S. Yang, M. O’Neill, M. A. Handschy, J. Soc. Info. Display 17, 369 (2009).

[3] M.A. Handschy, Display Devices 6, 8 (2007).

[4] E. H. Stupp, M.S. Brennesholtz, Projection Displays (Wiley, Chichester 1999).

[5] X.J. Yu, H.S. Kwok, Appl. Opt. 42, 6335 (2003).

[6] H.C. Huang, B. Zhang, H. Peng, H.S. Kwok, P.W. Cheng, Y.C. Chen, J. Soc. Info. Display 14, 499 (2006).

[7] V.G. Chigrinov, Liquid Crystal Devices: Physics and Applications (MA Artech House, Boston 1999).

[8] M.J. O’Callaghan, R. Ferguson, R. Vohra et al.,SID Symposium Digest 39, 232 (2008).

[9] E. Pozhidaev, V.C. Chigrinov, X.H. Li, Jpn. J. Appl. Phys. 45, 875 (2006).

[10] E. Pozhidaev,V.C. Chigrinov, T. Du, S. Kotova, M. Minchenko, V. Vashchenko, A. Krivosey, F. Fan, Proc. Eurodisplay Conference 41 (2009).

[11] X.H. Li, A. Murauski, A. Muravsky, P.Z. Xu, H. L. Cheung, V.G. Chigrinov, J. Display Techn. 3, 273 (2007).

[12] R. Kurihara, H. Furue, T. Takahashi, T. Yamashita, J. Xu, S. Kobayashi, Jpn. J. Appl. Phys. 40, 4622 (2001).

[13] S.T. Lagerwall, Ferroelectric and Antiferroelectric Liquid Crystals (Wiley-VCH, New York 1999). 